\title{
Respiratory Updates
}

\author{
Dr Farhana Mahmood, Specialty Doctor, Respiratory Medicine, RLI \\ Dr Timothy Gatheral, Consultant Respiratory Physician, RLI
}

\section{INTRODUCTION}

As with other fields, respiratory medicine has seen several significant advancements in recent years. The current status and prospects of certain noteworthy ones are presented.

\section{ADVANCES IN CYSTIC FIBROSIS TREATMENT}

Cystic fibrosis (CF) is an autosomal recessive disease caused by mutations in the $\mathrm{CF}$ transmembrane conductance regulator (CFTR) gene. The most common globally is F508del, however there are over 2000 variations reported, although not all cause disease. The subsequent CFTR protein defect causes abnormalities in both salt and fluid transport across epithelia, which in the lung, leads to dehydration of the airway surface and impaired mucociliary clearance (MCC). This failure of innate defence and mucus accumulation, possibly in conjunction with impaired bacterial killing, provides an undefended environment for opportunistic pathogens such as Pseudomonas aeruginosa, Staphylococcus aureus and Haemophilus influenzae. The host inflammatory response and subsequent tissue damage contribute to the characteristic decline in lung function as disease progresses. ${ }^{1}$

Conventional pulmonary treatment of CF has targeted the downstream consequences of the disease, namely mucus plugging and infection. The last few years have seen the emergence of more upstream therapeutic targets, several of which are in clinical trials, or have progressed to licensed treatments. ${ }^{1}$

\section{PRESENT MANAGEMENT STRATEGIES}

There are no known cures for cystic fibrosis. ${ }^{2}$ Management of $\mathrm{CF}$ is mainly through therapy including airway clearance, mucolytic drugs, bronchodilators, treatment of infections, anti-inflammatory therapy, pancreatic enzyme replacement, and bowel clearance. ${ }^{3}$ A major concern of CF is the manifestation of lung infections. For this, antibiotic therapy is carried out with particular regard to the microorganisms identified through sputum or throat $\mathrm{swab}^{3}$. In acute cases, in addition to the aforementioned, it may be necessary to provide respiratory support, including, non-invasive and invasive ventilation. ${ }^{4}$

\section{TREATMENTS TARGETING CFTR MUTATION}

Conventional treatment targets pathology driven CF and not the root cause. Drug research for therapies targeting CFTR action for the various classes of CFTR mutations are mainly focused on potentiators (which enhance the activity of correctly located CFTR channels), correctors (which correct defects on CFTR protein folding etc.) and read-through agents (which bypass premature ribosomal termination by codons to allow full-length CFTR production). ${ }^{1}$

\section{Potentiators}

The most significant advance in the treatment of CF over the last few years has been the development of ivacaftor (Kalydeco, Vertex USA). Trials have confirmed efficacy in Asp551Gly (G551D) the commonest mutation in this class and also more recently in rarer gating mutations. Significant improvements in $\mathrm{FEV}_{1}(\sim 10 \%$ absolute improvement), exacerbation rate, weight and healthrelated quality of life led to ivacaftor being licensed for use in these patients aged six years and above. ${ }^{1}$ Kalydeco is available for use in the United Kingdom (UK) for people with $\mathrm{CF}$ over the age of six months with one of nine rare gating mutations (G551D, G178R, S549N, S549R, G551S, G1244E, S1251N, S1255P or G139D), and to people aged 18 years and older who have the R117H mutation. It was demonstrated that ivacaftor achieves a CFTR activity equivalent to approximately $35 \%-40 \%$ of normal activity. ${ }^{6}$

\section{Correctors and combination therapy}

F508del is the commonest CF mutation globally. Lumacaftor (VX-809) restored CFTR function to around $15 \%$ of wild-type CFTR levels in vitro, but did not lead to significant clinical changes in F508del patients. ${ }^{6}$ Similarly, single-agent ivacaftor had shown little effect in this patient group, likely due to insufficient CFTR available at the cell surface for potentiation. ${ }^{7}$ Therefore the benefit of combined lumicaftor/ivacaftor treatment has been investigated. The phase III TRAFFIC and TRANSPORT trials showed significant improvements in FEV1, although this was of a lower magnitude (3-4\%) than seen in class III patients with ivacaftor and there was a substantial decrease in the number of pulmonary exacerbations in particular those leading to the requirement of IV antibiotics. ${ }^{8}$ Orkambi is available for use in the UK for people with CF over the age of two with two copies of the F508del mutation. Managed access agreements to make Orkambi available on the NHS have now been agreed in England, Scotland, Wales and Northern Ireland. ${ }^{9}$ The triple therapy of elexacaftor, tezacaftor, and ivacaftor has the potential to lead to "transformative improvements in the lives of people with cystic fibrosis," two phase III trials indicate. The researchers concluded, "elexacaftor plus tezacaftor plus ivacaftor provided clinically robust benefits compared with tezacaftor plus ivacaftor alone, with a favourable safety profile, and shows the potential to lead to transformative improvements in the lives of people with cystic fibrosis who are homozygous for the Phe508del mutation. ${ }^{10}$ Vertex has submitted the drug to the European Medicines Agency (EMA), who is analysing it for safety and clinical effectiveness. A decision is expected at the end of $2020 .{ }^{11}$

\section{COST}

It should be noted however that ivacaftor is currently a very high cost treatment (around \$294,000 per year), which is a significant issue for a lifelong therapy. ${ }^{6}$

Whilst insurance-based health care systems may be 
able to support the relatively small number of patients with CF suitable for these drugs, this is likely to pose a significant problem for nationalized health care systems. Strategies to tackle this, and the inevitable global inequalities which will arise, are urgently needed. ${ }^{1}$

Especially in Europe drug approval and licensing does not necessarily equate to drug availability; drug reimbursement is the competence of the national health authorities. Due to its high efficacy and despite its very high cost, reimbursement of Kalydeco has been granted in western European countries. ${ }^{12}$

NHS England has, however, very recently (Oct 2019) secured a definitive agreement with Vertex Pharmaceuticals to make available all three (Orkambi, Symkevi and Kalydeco) of their UK-licensed cystic fibrosis medicines. ${ }^{13}$

Around 3400 patients with CF are eligible for treatment with ivacaftor worldwide and this represents only around $5 \%$ of the total CF population. The benefits of ivacaftor on health and possibilities of active life, and the expected decrease in symptomatic treatment burden and hospitalisation (and their related costs) will need to be thoroughly assessed. ${ }^{6}$

\section{ADVANCES IN LUNG CANCER TREATMENT}

\section{Immunotherapy}

Non-small cell lung cancer (NSCLC) is a prevalent disease with high mortality and poor response to traditional cytotoxic therapy. Immunotherapeutic agents targeting immune checkpoint pathways have given promising results in clinical trials and are now being used in the management of advanced stage NSCLC..$^{14}$

Immune checkpoint inhibitors (ICIs) act by removing brakes on T-cell activation by antigen-presenting cells (APCs). The same principle may be used to cause T-cell attack on self-antigens which appear as a set of unique toxicities called immune-related adverse events (irAEs). ${ }^{14}$

ICIs are gradually being considered as useful in both early and late stage NSCLC due to having shown survival outcome improvement in patients with progressive NSCLC after traditional chemotherapy. ${ }^{14-16}$

Of late, the largest share of research interest for the development of ICIs is given to, among others, the programmed death-1 (PD-1 receptor), including its reciprocal ligands PD-L1 and PD-L2, and the cytotoxic T-lymphocyte antigen-4 (CTLA-4) pathways. ${ }^{14}$

Monoclonal antibodies targeting PD-1 (eg, nivolumab, pembrolizumab), CTLA-4 (ipilimumab), or PD-L1 (eg, durvalumab, atezolizumab, avelumab) have been studied in late phase clinical trials and demonstrated significant improvements in progression-free survival (PFS) and overall survival (OS) compared with secondline chemotherapy. These results show that such therapies are preferable in second-line for advanced NSCLC, and in some cases, first-line as well. ${ }^{14}$

Despite their purported benefits, ICIs carry a notable risk of toxicity including reports of colitis, hypophysitis, pneumonitis, thyroiditis, inflammatory arthritis, and more. ${ }^{14}$

\section{CLINICAL TRIALS}

\section{First-line setting}

Phase III trials using pembrolizumab (a PD1 ICI) monotherapy compared with standard platinum doublet chemotherapy in newly diagnosed NSCLC patients have shown improvement in PFS and OS. This study demonstrated the safety of co-administration of PD1 ICIs with traditional chemotherapy. ${ }^{17}$

\section{Stage III maintenance setting}

With the use of durvalumab (a PD-11 ICI), significant improvement in PFS (16.8 vs 5.6, with placebo) has been found in a phase III trial ${ }^{18}$ in patients with stage III unresectable NSCLC, after chemoradiation therapy (i.e. no evidence of disease progression after chemoradiation).

\section{Second-line setting}

Nivolumab (PD1 ICI) improved overall survival (nonsquamous: $39 \%-51 \%$ at 1 year; squamous: $24 \%-42 \%$ at 1 year) compared to single-agent docetaxel in patients with advanced non-squamous and squamous NSCLC who progressed on platinum-based regimens. ${ }^{19}$

\section{Incidence of checkpoint inhibitor pneumonitis}

Checkpoint inhibitor pneumonitis (CIP) is an irAE for which incidence rates as high as $13 \%$ have been reported in phase I trials of ICIs in NSCLC. ${ }^{20}$ However, in large clinical trials and meta-analyses this is around 3-5\%. ${ }^{21}$ In some earlier cases, it is possible that patients' symptoms may have been reported as dyspnoea, cough or chest pain, without a diagnosis of CIP. Incidence appears to vary with the type of ICI being used, with reports indicating higher occurrence of pneumonitis in patients treated with anti-PD-1 therapy than those treated with anti-PD-L1 therapy. Combination and/or concurrent ICI therapy may have an effect on CIP rates as observed in a recent phase III trial..$^{14,18}$

\section{Clinical presentation of CIP}

Symptoms of patients with CIP is nonspecific and may include dyspnoea, cough, fever, chest pain, and progressing decrease in exercise tolerance. Time of onset is very variable with a reported median time to onset of 2.8 months from a large retrospective review of CIP in patients receiving anti-PD-1/PD-L1 ICIs. ${ }^{21}$ Severity of CIP tends to increase from after between 100 and 200 days following start of therapy. ${ }^{22}$ For early detection of CIP, the following entities should be considered in the differential: infection (most patients should undergo bronchoscopy to exclude this), pneumocystis pneumonia, fulminant myocarditis, radiation pneumonitis, alveolar haemorrhage and active tuberculosis. ${ }^{14}$

\section{Management}

Trials made so far have not been aimed at deciding on best treatment strategies for patients with CIP, with current guidelines and recommendations based on small reports and case series. Corticosteroid therapy is the dominant means of management; typically high doses of $1-4 \mathrm{mg} / \mathrm{hg}$ 
are initiated following diagnosis. ${ }^{14}$ Current guidelines ${ }^{23}$ recommend a dose of $1 \mathrm{mg} / \mathrm{kg} / \mathrm{d}$ of prednisone for lowergrade (ie, grade 2) CIP and $2-4 \mathrm{mg} / \mathrm{kg} / \mathrm{d}$ for higher-grade (ie, grade 3-4) CIP. Patients who remain without clinical improvement after 48 to 72 hours of corticosteroids are considered steroid- refractory.

\section{EXPANDING ROLE OF ANTI-FIBROTICS IN INTERSTITIAL LUNG DISEASE}

Interstitial Lung Disease (ILD) describes a collection of over 200 parenchymal lung disorders, including diseases without known causes called idiopathic interstitial pneumonias (IIPs). The majority of these disorders are considered rare. Idiopathic pulmonary fibrosis (IPF) is an ILD that is most widely studied among others. ${ }^{24}$ This disease is found to affect mostly adults aged over 60 years. Symptoms include progressive pulmonary fibrosis, decline in lung function and high mortality rate. Some patients with other types of ILDs also develop a progressive fibrosing phenotype with similarities in behaviour to $\mathrm{IPF}^{24}$ Examples of such ILDs include connective tissue disease-related ILDs (CTD-ILDs) such as those related to rheumatoid arthritis (RA-ILD), systemic sclerosis (SScILD), and polymyositis/dermatomyositis; ILD related to chronic sarcoidosis; chronic hypersensitivity pneumonitis (HP); idiopathic non-specific interstitial pneumonia (iNSIP) and unclassifiable ILD ${ }^{24}$. Due to the overlapping clinical, radiological and pathological presentations, these ILDs are generally all termed as progressive-fibrosing ILDs (PF-ILD). It has been suggested that the fibrosis is diagnosed as "progressive", when, within a 24-month period any of the following is observed: decline of $\geqslant 10 \%$ in forced vital capacity (FVC); a relative decline of $\geqslant 15 \%$ in diffusing capacity of the lung for carbon monoxide (DLCO); or worsening symptoms or a worsening radiological appearance accompanied by $a \geqslant 5-<10 \%$ relative decrease in FVC. ${ }^{25}$

\section{Symptoms}

Progression of fibrosing ILD is characterised by a decline in lung function, worsening of symptoms, deterioration in health-related quality of life, and high mortality rate. Disease progression is most commonly assessed through measurement of forced vital capacity (FVC) and diffusion capacity of the lungs for carbon monoxide (DLco). ${ }^{24}$

\section{INBUILD® study}

The progressive fibrosis of the lung leads to an irreversible loss of lung function and is associated with high morbidity and mortality. On average, $18-32 \%$ of patients with ILD might develop a progressive pulmonary fibrosis. ${ }^{26}$ Progressive fibrosing interstitial lung diseases encompass a range of clinical diagnoses, including: hypersensitivity pneumonitis, sarcoidosis, autoimmune ILDs such as rheumatoid arthritis-associated ILD, systemic sclerosisassociated ILD, mixed connective tissues disease-associated ILD, idiopathic non-specific interstitial pneumonia, and unclassified idiopathic interstitial pneumonia amongst others $^{25}$.
Patients with progressive fibrosing interstitial lung diseases are a neglected patient population for whom no approved treatment options exist that effectively influence the course of their disease. ${ }^{26}$

Nintedanib (Ofev, Boehringer Ingelheim, Germany) is one of two antifibrotic drugs shown to slow the disease progression in IPF, and they are approved and recommended for use in IPF patients by international guidelines. ${ }^{27}$ In September 2019, nintedanib was approved in the U.S. as the first and only therapy to slow the rate of decline in pulmonary function in patients with systemic sclerosis-associated ILD. ${ }^{26}$ Nintedanib is already approved in more than 70 countries for the treatment of patients living with idiopathic pulmonary fibrosis (IPF) - a chronic and ultimately fatal disease characterised by a decline in lung function. It is estimated that over 80,000 people with IPF have been treated with nintedanib. ${ }^{26}$

The INBUILD trial was a randomised, double-blind, placebo-controlled, parallel group trial conducted at 153 sites in 15 countries that evaluated the efficacy, safety, and tolerability of nintedanib (150 mg, 2 x daily) over 52 weeks in patients with progressive fibrosing interstitial lung disease. Eligible patients were aged $\geq 18$ years with a physician-diagnosed ILD other than IPF and features of fibrosing lung disease of $>10 \%$ extent in high-resolution computed tomography (HRCT). ${ }^{26}$ Patients were required to meet criteria for ILD progression within 24 months before screening, based on decline in FVC, increased fibrotic changes on imaging, or worsening of symptoms, despite treatment with drugs commonly used in clinical practice to treat ILD. ${ }^{26}$

A total of 663 patients, of whom $412(62.1 \%)$ had a usual interstitial pneumonia (UIP) fibrotic pattern on HRCT, were randomised 1:1 to receive oral nintedanib 150 $\mathrm{mg}$ twice daily or placebo. The primary endpoint was the annual rate of decline in FVC (mL/year) assessed over 52 weeks. FVC is a lung function test measuring the amount of air which can be forcibly exhaled from the lungs after taking the deepest breath possible. As fibrosis progresses, lung function gradually and irreversibly deteriorates. ${ }^{26}$

In the INBUILD trial, nintedanib slowed lung function decline by $57 \%$ across the overall study population, with an adjusted annual rate of decline over 52 weeks in FVC of -80.8 mL/year compared to -187.8 $\mathrm{mL} /$ year for placebo (difference, $107.0 \mathrm{~mL} /$ year $[95 \%$ CI, 65.4 to 148.5 ]; $\mathrm{p}<0.001$ ). Nintedanib demonstrated a consistent effect on lung function decline in patients with an usual interstitial pneumonia (UIP) like fibrotic pattern and those with other fibrotic patterns on HRCT. The most common adverse event was diarrhoea, reported in $66.9 \%$ and $23.9 \%$ of patients treated with nintedanib and placebo, respectively. The safety profile observed in INBUILD was consistent to what has been seen in IPF and SSc-ILD patients treated with nintedanib previously. ${ }^{26}$

The study has met its primary endpoint and demonstrated the efficacy and safety of nintedanib in patients with a broad range of progressive fibrosing interstitial lung diseases other than idiopathic pulmonary fibrosis (IPF). Nintedanib was shown to slow the rate of ILD progression independent of the fibrotic pattern seen on chest imaging. The side effect profile was consistent with previous studies of nintedanib in ILDs, with diarrhoea being the most common adverse event. ${ }^{26}$ 


\section{REFERENCES}

1. Edmondson C, Davies JC. Current and future treatment options for cystic fibrosis lung disease: latest evidence and clinical implications. Therapeutic Advances in Chronic Disease. 2016;7:170-83.

2. Massie J, Delatycki MB. Cystic Fibrosis Carrier Screening. Paediatric Respiratory Reviews. 2013;14:270-5.

3. Goldman LeeS Andrew I. Goldman-cecil Medicine. Elsevier - Health Science; 2011.

4. Lawrence H, Moore T, Usmani O, Hickin S. Respiratory system. 2019.

5. Cystic Fibrosis Trust. Kalydeco [Internet]. Available from: https://www.cysticfibrosis.org.uk/the-work-we-do/ campaigning-hard/life-saving-drugs/kalydeco

6. Fajac I, De Boeck K. New horizons for cystic fibrosis treatment. Pharmacology \& Therapeutics. 2017;170:20511 .

7. Aitken ML, Bellon G, De Boeck K, Flume PA, Fox HG, Geller DE, et al. Long-Term Inhaled Dry Powder Mannitol in Cystic Fibrosis: An International Randomized Study. Am J Respir Crit Care Med. 2012;185:645-52.

8. Davies JC, Wainwright CE, Canny GJ, Chilvers MA, Howenstine MS, Munck A, et al. Efficacy and Safety of Ivacaftor in Patients Aged 6 to 11 Years with Cystic Fibrosis with a G551D Mutation. Am J Respir Crit Care Med. 2013;187:1219-25.

9. Cystic Fibrosis Trust. Orkambi [Internet]. Available from: https://www.cysticfibrosis.org.uk/the-work-we-do/ campaigning-hard/life-saving-drugs/orkambi

10. Mahase E. Cystic fibrosis: triple therapy shows promising results. BMJ. 2019;16347.

11. Cystic Fibrosis Trust. Triple combination therapy (Trikafta) [Internet]. Available from: https://www.cysticfibrosis.org. uk/the-work-we-do/campaigning-hard/life-saving-drugs/ triple-combination-therapy

12. De Boeck K, Davies JC. Where are we with transformational therapies for patients with cystic fibrosis? Current Opinion in Pharmacology. 2017;34:70-5.

13. NHS England. NHS England concludes wide-ranging deal for cystic fibrosis drugs [Internet]. Available from: https:// www.england.nhs.uk/2019/10/nhs-england-concludeswide-ranging-deal-for-cystic-fibrosis-drugs/

14. Suresh K, Naidoo J, Lin CT, Danoff S. Immune Checkpoint Immunotherapy for Non-Small Cell Lung Cancer. Chest. 2018;154:1416-23.

15. Brahmer JR, Tykodi SS, Chow LQM, Hwu W-J, Topalian SL, Hwu P, et al. Safety and Activity of Anti-PD-L1 Antibody in Patients with Advanced Cancer. N Engl J Med. 2012;366:2455-65.

16. Borghaei H, Paz-Ares L, Horn L, Spigel DR, Steins M, Ready NE, et al. Nivolumab versus Docetaxel in Advanced Nonsquamous Non-Small-Cell Lung Cancer. N Engl J Med. 2015;373:1627-39.

17. Reck M, Rodríguez-Abreu D, Robinson AG, Hui R, Csőszi T, Fülöp A, et al. Pembrolizumab versus Chemotherapy for PD-L1-Positive Non-Small-Cell Lung Cancer. N Engl J Med. 2016;375:1823-33.

18. Antonia SJ, Villegas A, Daniel D, Vicente D, Murakami $S$, Hui R, et al. Durvalumab after Chemoradiotherapy in Stage III Non-Small-Cell Lung Cancer. N Engl J Med. 2017;377:1919-29.

19. Larkin J, Chiarion-Sileni V, Gonzalez R, Grob JJ, Cowey CL, Lao CD, et al. Combined Nivolumab and Ipilimumab or Monotherapy in Untreated Melanoma. N Engl J Med. 2015;373:23-34.

20. Nishino M, Giobbie-Hurder A, Hatabu H, Ramaiya NH, Hodi FS. Incidence of Programmed Cell Death 1 Inhibitor-Related Pneumonitis in Patients With Advanced Cancer: A Systematic Review and Meta-analysis. JAMA Oncol. 2016;2:1607.
21. Naidoo J, Wang X, Woo KM, Iyriboz T, Halpenny D, Cunningham J, et al. Pneumonitis in Patients Treated With Anti-Programmed Death-1/Programmed Death Ligand 1 Therapy. JCO. 2017;35:709-17.

22. Voong KR, Hazell S, Hu C, Hayman J, Hales R, Marrone $\mathrm{K}$, et al. MA 09.08 Receipt of Chest Radiation and ImmuneRelated Pneumonitis in Patients with NSCLC Treated with Anti-PD-1/PD-L1. Journal of Thoracic Oncology. 2017;12:S1837.

23. Haanen J, Carbonnel F, Robert C, Kerr K, Peters S, Larkin $\mathrm{J}$, et al. Management of toxicities from immunotherapy: ESMO Clinical Practice Guidelines for diagnosis, treatment and follow-up. Annals of Oncology. 2017;28:iv119-42.

24. Kolb $M$, Vašáková $M$. The natural history of progressive fibrosing interstitial lung diseases. Respir Res. 2019;20:57.

25. Cottin V, Hirani NA, Hotchkin DL, Nambiar AM, Ogura $T$, Otaola $M$, et al. Presentation, diagnosis and clinical course of the spectrum of progressive-fibrosing interstitial lung diseases. Eur Respir Rev. 2018;27:180076.

26. Boehringer Ingelheim. INBUILD ${ }^{\circledR}$ meets primary endpoint - study evaluated nintedanib in patients across a range of progressive fibrosing interstitial lung diseases [Internet]. 2019. Available from: https://www.boehringer-ingelheim. $\mathrm{com} /$ press-release/pfildinbuildtrialmeetsprimaryendpoint

27. Flaherty KR, Brown KK, Wells AU, Clerisme-Beaty E, Collard HR, Cottin V, et al. Design of the PF-ILD trial: a double-blind, randomised, placebo-controlled phase III trial of nintedanib in patients with progressive fibrosing interstitial lung disease. BMJ Open Resp Res. 2017;4:e000212.

Correspondence to: farhana.mahmood@mbht.nhs.uk

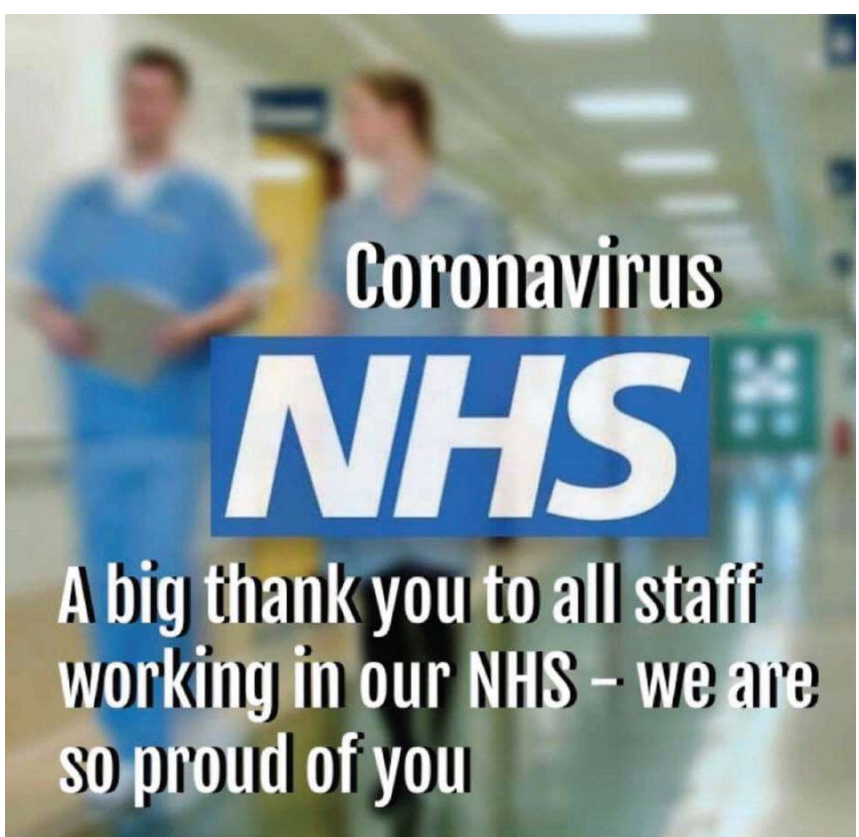

